\title{
Solution NMR assignment of the ARC4 domain of human tankyrase 2
}

\author{
Mariola Zaleska ${ }^{1} \cdot$ Katie Pollock ${ }^{1,2} \cdot$ Ian Collins ${ }^{2} \cdot$ Sebastian Guettler ${ }^{1} \cdot$ Mark Pfuhl $^{3} \mathbb{C}$
}

Received: 8 February 2019 / Accepted: 2 March 2019 / Published online: 7 March 2019

(c) The Author(s) 2019

\begin{abstract}
Tankyrases are poly(ADP-ribose)polymerases (PARPs) which recognize their substrates via their ankyrin repeat cluster (ARC) domains. The human tankyrases (TNKS/TNKS2) contain five ARCs in their extensive N-terminal region; of these, four bind peptides present within tankyrase interactors and substrates. These short, linear segments, known as tankyrasebinding motifs (TBMs), contain some highly conserved features: an arginine at position 1, which occupies a predominantly acidic binding site, and a glycine at position 6 that is sandwiched between two aromatic side chains on the surface of the ARC domain. Tankyrases are involved in a multitude of biological functions, amongst them $\mathrm{Wnt} / \beta$-catenin signaling, the maintenance of telomeres, glucose metabolism, spindle formation, the DNA damage response and Hippo signaling. As many of these are relevant to human disease, tankyrase is an important target candidate for drug development. With the emergence of non-catalytic (scaffolding) functions of tankyrase, it seems attractive to interfere with ARC function rather than the enzymatic activity of tankyrase. To study the mechanism of ARC-dependent recruitment of tankyrase binders and enable protein-observed NMR screening methods, we have as the first step obtained a full backbone and partial side chain assignment of TNKS2 ARC4. The assignment highlights some of the unusual structural features of the ARC domain.
\end{abstract}

Keywords ADP-ribosylation · Ubiquitylation · Ankyrin repeats · Signaling

\section{Biological context}

The tankyrases (TNKS/ARTD5, TNKS2/ARTD6) are poly(ADP-ribose)polymerases (PARPs) and as such catalyse the processive modification of protein substrates with poly(ADP-ribose) (PAR) chains, thereby consuming their co-substrate $\mathrm{NAD}^{+}$(Haikarainen et al. 2014). PARPs are part of a larger family of Diphtheria-toxin-like ADP-ribosyltransferases (ARTDs), which share variants of a conserved catalytic domain that either modifies substrates with monoADP-ribose or PAR, or lacks detectable catalytic activity (Hottiger et al. 2010; Vyas et al. 2014). Different ARTD family members are distinguished by unique combinations

Mark Pfuhl

mark.pfuhl@kcl.ac.uk

1 Divisions of Structural Biology \& Cancer Biology, The Institute of Cancer Research (ICR), London SW7 3RP, UK

2 Division of Cancer Therapeutics, The Institute of Cancer Research (ICR), London SW7 3RP, UK

3 School of Cardiovascular Medicine and Sciences and Randall Centre, King's College London, Guy's Campus, London SE1 1UL, UK of accessory domains, which confer functional diversity (Hottiger et al. 2010). In the case of the tankyrases, these are an extensive $\mathrm{N}$-terminal region comprising five consecutive ankyrin repeat clusters (ARCs), either flexibly or rigidly linked, and responsible for substrate recruitment (Seimiya et al. 2004; Guettler et al. 2011; Eisemann et al. 2016). The ARCs are followed by a polymerizing sterile alpha motif (SAM) domain (De Rycker and Price 2004; Mariotti et al. 2016; Riccio et al. 2016) that precedes the PARP domain (Lehtiö et al. 2008). ARCs and the SAM domain direct tankyrase to regulators of a wide range of biological processes, among which Wnt/ $\beta$-catenin signaling (Huang et al. 2009; Mariotti et al. 2016, 2017; Yang et al. 2016), the maintenance and mitotic resolution of telomeres (Smith et al. 1998; Smith and de Lange 2000; Dynek and Smith 2004) and glucose metabolism (Chi and Lodish 2000; Yeh et al. 2007; Zhong et al. 2016) are some of the best-studied. Additional roles of tankyrase include the regulation of mitotic spindle formation (Chang et al. 2005, 2009), Hippo signaling (Wang et al. 2015; Troilo et al. 2016; Jia et al. 2017) and emerging functions in the DNA damage response (Nagy et al. 2016), cell migration (Lupo et al. 2016), and Notch signaling (Bhardwaj et al. 2017), to name a few. Proteomics 
studies and in-silico predictions of tankyrase binders illustrate the diverse cellular functions of tankyrase (Guettler et al. 2011; Li et al. 2017; Bhardwaj et al. 2017).

Of the five ARCs, four (ARCs 1, 2, 4 and 5) are known to bind substrates featuring a degenerate six- to eight-aminoacid peptide motif known as the tankyrase-binding motif (TBM); ARC3 shows no detectable substrate binding (Sbodio and Chi 2002; Seimiya et al. 2004; Guettler et al. 2011). Studies reported in 2011 and 2012 first revealed the structural basis of substrate recruitment by tankyrase (Guettler et al. 2011; Morrone et al. 2012). To date, numerous additional crystal structures of tankyrase ARCs from both TNKS and TNKS2, typically complexed with TBM peptides, are available (Li et al. 2016; Eisemann et al. 2016; Xu et al. 2017a, b; DaRosa et al. 2018).

The TBM binding site in substrate-binding ARCs is highly conserved (Guettler et al. 2011). It features a subpocket, the so-called 'arginine cradle', that engages an essential arginine residue at TBM position 1. A second key binding determinant is a channel lined by two aromatic amino acids which accommodates an essential glycine residue at TBM position 6, forming an 'aromatic glycine sandwich' (Guettler et al. 2011). Their strict requirement suggests that the arginine and glycine residues represent critical docking points in substrate recruitment. Additional contacts are provided by residues at TBM positions 4 and 5, which interact with a 'central patch' in the peptide-binding pocket, and at TBM position 8 , where an acidic residue can confer increased binding affinity through a salt bridge (Guettler et al. 2011). The eight amino acids of the TBM do not need to be contiguous: the essential arginine can be $\mathrm{N}$-terminally displaced to varying extent, and structural plasticity or looping of such TBM peptides enables both the arginine and a hydrophobic side chain at position 4 to dock as in "canonical" TBM peptides (Morrone et al. 2012; DaRosa et al. 2018). Moreover, there is evidence for tankyrase targets devoid of detectable TBM sequences ( $\mathrm{Li}$ et al. 2017; Bhardwaj et al. 2017). These binders may either indirectly interact with tankyrase or be recruited through direct binding by alternative, hitherto unknown binding mechanisms.

Tankyrases are being explored as potential therapeutic targets in conditions such as cancer, neurodegeneration, fibrosis and diabetes (Riffell et al. 2012; Haikarainen et al. 2014; Zhong et al. 2016; Mariotti et al. 2017). These efforts led to the development of a wide range of tankyrase catalytic inhibitors (Haikarainen et al. 2014; Mariotti et al. 2017). The response of tankyrase to catalytic inhibition, however, is complex. It not only leads to the loss of tankyrase's enzymatic PARP activity but typically also to tankyrase accumulation and that of many of its substrates (Huang et al. 2009; Zhang et al. 2011; Bhardwaj et al. 2017) and potentially to increased tankyrase polymerization (De Rycker and Price 2004). The accumulation of tankyrase and its substrates upon tankyrase catalytic inhibition is a consequence of an attenuated PAR-dependent ubiquitination pathway (Zhang et al. 2011; DaRosa et al. 2015). Moreover, some activities of tankyrase may be mediated by non-catalytic (scaffolding) mechanisms: at least elevated tankyrase levels can drive Wnt/ $\beta$-catenin signalling independently of PARylation, requiring only the substrate-binding ARCs and the polymerizing SAM domain (Mariotti et al. 2016). Consequently, targeting tankyrase's accessory domains provides an attractive alternative means to inhibit tankyrase function. This approach would block both tankyrase-dependent scaffolding and substrate PARylation. As proof of concept, a recent study demonstrates that an affinity-optimised TBM (Guettler et al. 2011), when stabilised by cyclisation and fused to a facilitator of cell permeability, can inhibit $\mathrm{Wnt} / \beta$-catenin signalling in cells (Xu et al. 2017b).

Here, we report the assignment of ARC4 of human TNKS2, which will enable a more elaborate characterisation of substrate recruitment by tankyrase ARCs and the development of substrate binding antagonists.

\section{Methods and experiments}

The expression construct for TNKS2 ARC4 (NM_025235), comprising residues 488-649 cloned into vector pETM-30, was described previously (Guettler et al. 2011). To produce ${ }^{15} \mathrm{~N}$-labelled or ${ }^{15} \mathrm{~N}-{ }^{13} \mathrm{C}$-labelled protein, E. coli (BL21CodonPlus(DE3)-RIL) cells were grown in M9 minimal media (Laboratory Support Services, ICR) supplemented with ${ }^{15} \mathrm{NH}_{4} \mathrm{Cl}$ or ${ }^{15} \mathrm{NH}_{4} \mathrm{Cl}$ (at $1 \mathrm{~g} / \mathrm{L}$ M9) and ${ }^{13} \mathrm{C}$ D-glucose (Cambridge Isotope Laboratories) (at $6 \mathrm{~g} / \mathrm{L}$ M9), respectively, following the protocol of Marley and colleagues (Marley et al. 2001). Briefly, freshly transformed bacteria were grown at $37^{\circ} \mathrm{C}$ in $4 \mathrm{~L}$ of standard LB media (Laboratory Support Services, ICR) containing kanamycin (50 $\mu \mathrm{g} /$ $\mathrm{mL})$ and chloramphenicol $(34 \mu \mathrm{g} / \mathrm{mL})$ until they reached an $\mathrm{OD}_{600}$ of 0.7. At this point, cells were transferred into M9 minimal media containing appropriate isotope(s) by collecting cells by centrifugation and resuspending them in $1 \mathrm{~L}$ of the final M9 minimal media. The culture was incubated at $37{ }^{\circ} \mathrm{C}$ for $1 \mathrm{~h}$ to allow the cells to recover, and protein expression was induced by addition of $0.5 \mathrm{mM}$ IPTG. Protein expression was carried out at $18{ }^{\circ} \mathrm{C}$ for $16 \mathrm{~h}$. Cells were next harvested by centrifugation, and the pellet was stored at $-80{ }^{\circ} \mathrm{C}$ until purification following the previously described method (Guettler et al. 2011; Pollock et al. 2017).

NMR samples were prepared in a buffer of $20 \mathrm{mM}$ sodium phosphate $\mathrm{pH} 7.0,100 \mathrm{mM}$ sodium chloride and $1 \mathrm{mM}$ TCEP with a protein concentration of $1 \mathrm{mM}$. Backbone and partial sidechain assignments of the domain were obtained from a combination of 3D HNCACB, $(\mathrm{H})$ $\mathrm{C}(\mathrm{CCO}) \mathrm{NH}, \mathrm{HNCO}, \mathrm{HN}(\mathrm{CA}) \mathrm{CO}$ and ${ }^{15} \mathrm{~N}$ resolved $3 \mathrm{D}$ 
NOESY-HSQC experiments recorded at 700 and $800 \mathrm{MHz}$ on Bruker Avance spectrometers at $20^{\circ} \mathrm{C}$. Spectra were processed with Topspin 3.1 (Bruker), and all assignments were performed with CCPN analysis 2.4 (Vranken et al. 2005).

\section{Assignments and data deposition}

The TNKS2 ARC4 domain construct comprises 165 amino acids (residues 488-649 plus 3 additional N-terminal amino acids, GAM, resulting from the cloning method) and despite its substantial molecular weight of $17.8 \mathrm{kDa}$ gives excellent NMR spectra (see Fig. 1). It was possible to find assignments for 164 residues with only the $\mathrm{N}$-terminal glycine missing completely. Of the assigned residues, backbone amide peaks were missing for only two of the non-proline residues (R525 and V584). Out of a total of 165 backbone nitrogens, $165 \alpha$ carbons, $151 \beta$ carbons, $140 \gamma$ carbons, $99 \delta$ carbons, $31 \varepsilon$ carbons and 165 backbone carbonyls, a total of 156 (94.5\%), 163 (98.7\%), 148 (98.0\%), 68 (48.6\%), $42(42.4 \%)$ and 11
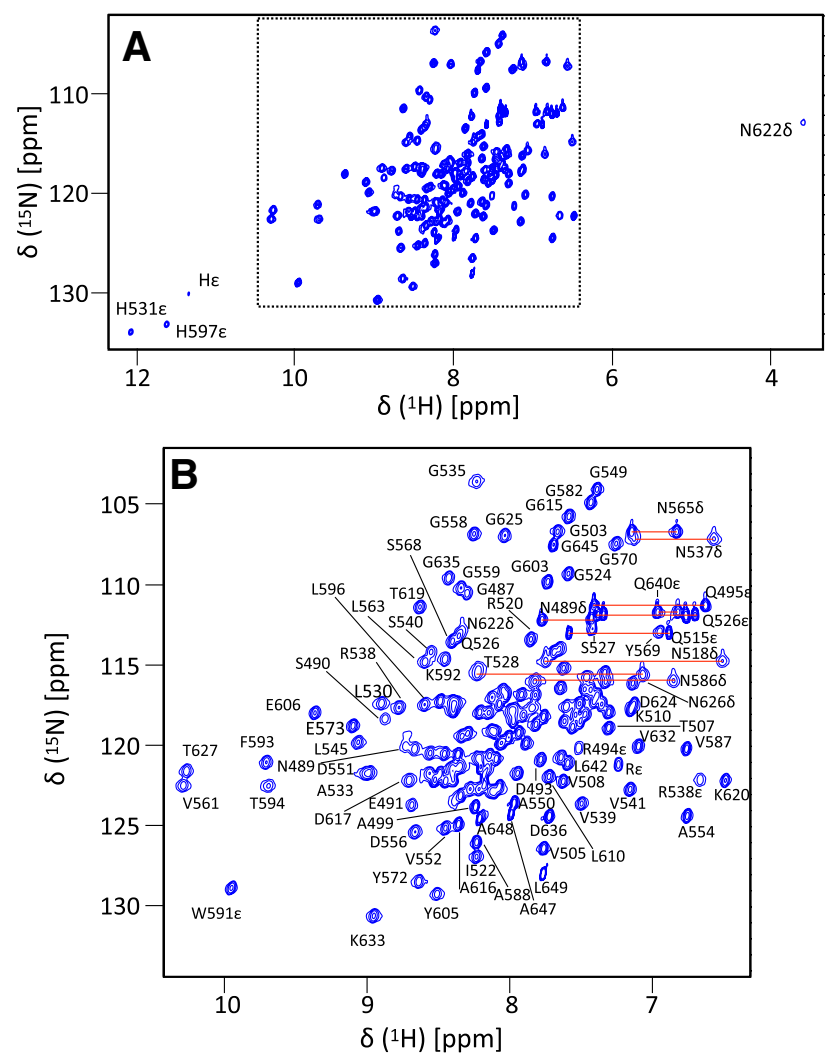

Fig. $1{ }^{1} \mathrm{H}_{-}{ }^{15} \mathrm{~N}$ HSQC spectrum of $1 \mathrm{mM}$ uniformly ${ }^{15} \mathrm{~N} /{ }^{13} \mathrm{C}$ labelled ARC4 recorded at a temperature of $293 \mathrm{~K}$ and a field of $700 \mathrm{MHz}$. Note that the sidechain resonances of histidines and arginines are folded from their original position in the ${ }^{15} \mathrm{~N}$ dimension. a Overview spectrum. b Majority of the backbone resonances in the spectrum (indicated by box in a). Well resolved peaks have been labelled with their assignments; pairs of peaks for sidechain $\mathrm{NH}_{2}$ groups are connected by red lines
(35.4\%), respectively, could be assigned. The assignment has been deposited with the BMRB, accession code 27747 .

\section{Comparison to X-ray structure}

The NMR spectra of ARC4 contain a number of unusual features, most prominently the appearance of 3 peaks for histidine sidechain $\mathrm{N} \varepsilon 2 / \mathrm{H} \varepsilon 2$ groups, two of which could be assigned. Such resonances are usually exchange-broadened beyond detection. ARC4, however, makes a rather unusual use of histidines as part of the conserved ankyrin repeat (AR) infrastructure, with regular occurrences at the N-terminal end of the first helix and the C-terminal end of the second helix of each AR. Thus, they appear on opposite sides of the protein in the central three ARs (see Fig. 2). Those at the C-termini of the second helix point into solution whereas those at the $\mathrm{N}$-termini of the first are covered by the long loop/beta hairpins which connect adjacent ARs. The latter are part of a highly conserved TPLH sequence motif (Mosavi et al. 2002). As part of this motif, the histidine $\mathrm{H} \varepsilon 2$ acts as an $\mathrm{H}$-bond donor to the backbone carbonyl group of the residue preceding the TPLH motif in the following repeat (Preimesberger et al. 2015). Our observation of three histidine sidechain $\mathrm{N} \varepsilon 2 / \mathrm{H} \varepsilon 2$ groups is therefore in good agreement with the structure. Curiously, the $\mathrm{C}_{\alpha}$ secondary shifts of two of these three protected histidines (H531 with almost 9 ppm, H564 with almost 10 ppm; H597

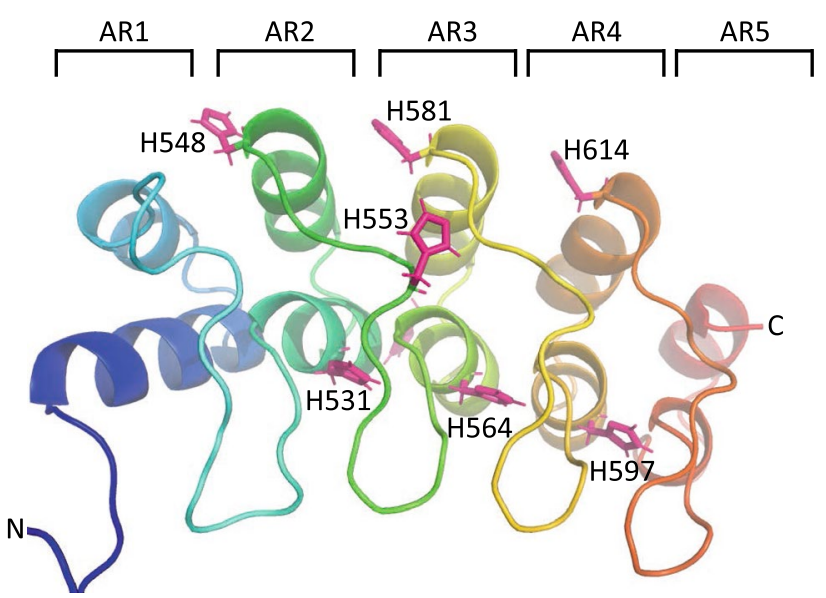

Fig. 2 Structure of ARC4 (PDB code 3TWQ) showing all histidine side chains as sticks in pink while the main backbone is shown in cartoon style coloured by sequence from $\mathrm{N}$-terminus (blue, left) to C-terminus (red, right). Ankyrin repeats (ARs) are numbered AR1 to AR5. The positions of the histidines occupying key positions in the three central ARs are clearly visible with H548, H581 and H614 pointing towards the solvent (top) while H531, H564 and H597 (bottom) are covered by the $\beta$-hairpins linking ARs. H553 (front) and H571 (back, not labelled) are not part of the conserved AR pattern and exposed to solvent 

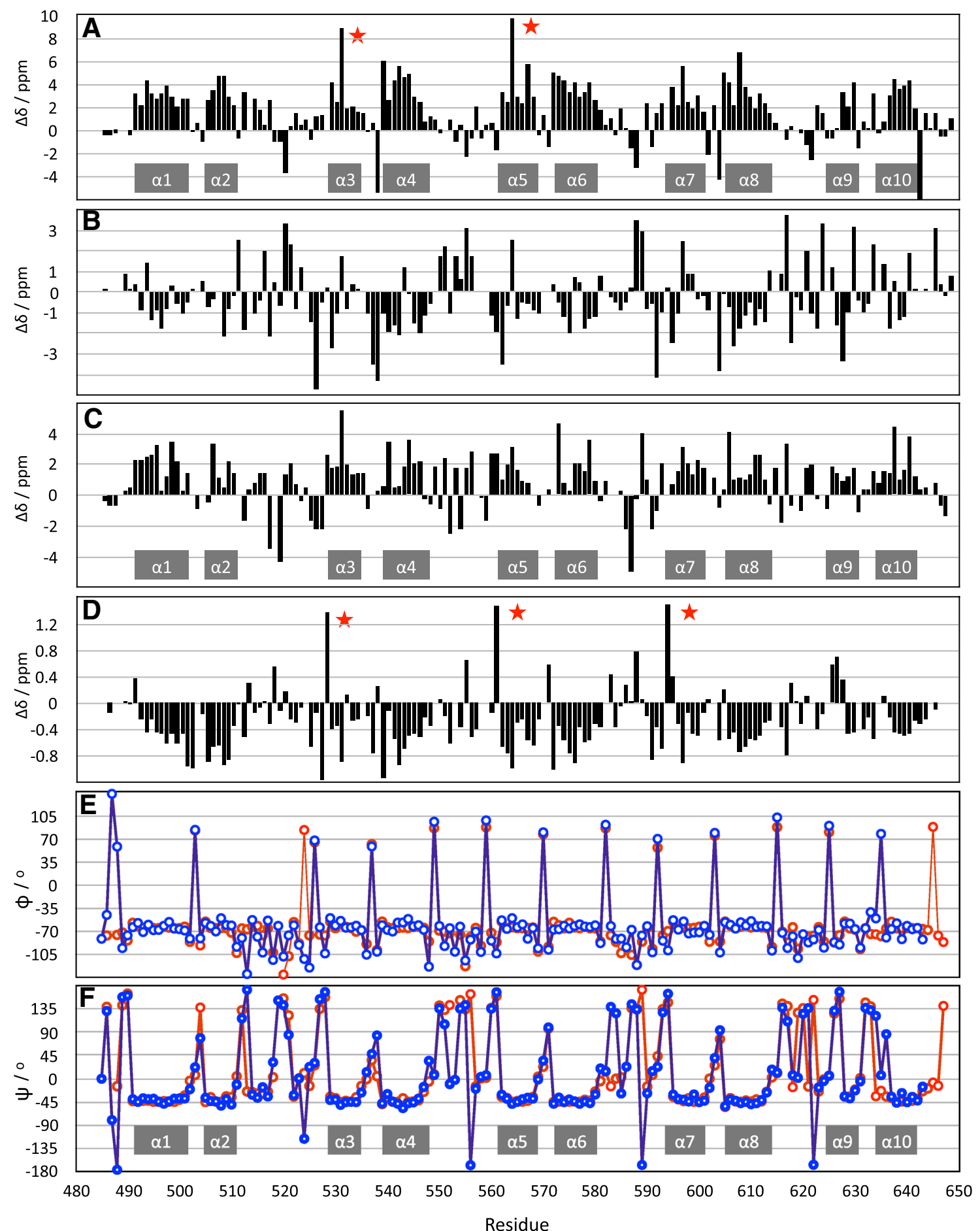

Fig. 3 Secondary chemical shifts (Wishart and Sykes 1994) and backbone dihedral angles predicted from Dangle and extracted from the crystal structure of ARC4 (PDB code 3TWQ) (Guettler et al. 2011) All values were calculated using CCPN analysis version 2.4, and figures were generated in Apple Numbers, inkscape and keynote.

with $5.5 \mathrm{ppm}$ is less extreme but still substantial) are well outside the $[-5.0,+5.0]$ bracket of all the other residues (see Fig. 2). This pattern is less apparent for the $C_{\beta}$ and a $\mathrm{C} \alpha$, b $\mathrm{C} \beta$, c C', d H $\alpha$, e phi, f psi. Dihedral angles from Dangle are shown in red, those from the crystal structure in blue. Positions of secondary structure elements based on the chemical shift analysis are indicated as grey bars. Positions of the protected histidines with chemical shift outliers are indicated by red stars

C' secondary shifts. However, it is repeated, albeit with a shift of -3 in the sequence, for the $\mathrm{H}_{\alpha}$ secondary chemical shifts: residues T528, V561 and T594 have values of around 
+1.4 , much larger than all the other values, which are well within the $[-0.8,+0.8 \mathrm{ppm}]$ bracket. It is not completely clear what causes the unusual chemical shifts. The fact that these instances of unusual secondary chemical shifts occur in precisely repeated structural units in AR-AR boundaries suggests that the most unusual backbone conformation, in combination with the hydrogen bonds from the histidine side chains to the backbone amides i-3 (Preimesberger et al. 2015), are likely to be the causative factor.

Another unusual feature is the sidechain amide group of N622, which has proton resonances at 8.33 and $3.59 \mathrm{ppm}$. The extreme shift of one of the amides can be explained by its position extremely close to the aromatic ring of F593, with which it is very likely to form a $\pi$-hydrogen bond.

Finally, we can observe three arginine sidechain $\mathrm{N} \varepsilon$, which is also not common at $\mathrm{pH}$ values around 7. Two of these could be assigned to R538 and R494. R494 is very likely involved in simultaneous salt bridges with the neighbouring E491 and E523 (shortest distances from

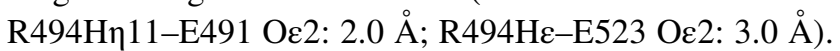
The He of R538 is likely to make a hydrogen bond to the backbone carbonyl oxygen of K501 (distance R538 He-K501 O’: $2.0 \AA$ ).

\section{Secondary structure}

We analysed the secondary structure of ARC4, based on the backbone chemical shifts, and compared it to the crystal structure (Guettler et al. 2011) (PDB code 3TWQ). In the first instance, we qualitatively compared the positions of the helices; we next quantitatively compared the backbone dihedral angles predicted by Dangle (Cheung et al. 2010) to those extracted from the crystal structure (see Fig. 3). The positions of the helices are generally in good agreement with the individual secondary chemical shifts, especially the $\mathrm{C}_{\alpha}$ and $\mathrm{H}_{\alpha}$ values, while $\mathrm{C}^{\prime}$ and $\mathrm{C}_{\beta}$ provide a less clear correlation. The only exception is helix 9 where some values for $\mathrm{H}_{\alpha}$ deviate somewhat. Even more intriguing is the result of the Dangle analysis. We can see an excellent agreement of the values with those from the crystal structure. Most importantly, the sharp changes of phi and psi between the helices are precisely matched for most residues. (Note the apparently huge difference in psi prior to the first helix of each AR (H1, H3, H5, H7, H9), which is actually very small due to the circular periodicity of the dihedral value; i.e., a value of $+175^{\circ}$ is actually very close to $-175^{\circ}$.) The only deviations are seen prior to helix 3, near to H531 and at the $\mathrm{C}$-terminus. The region around the former folds in an unusual way and involves rare interactions which cause unusual chemical shifts as outlined above. At the latter, the conformation is less well defined and likely to differ between solution and crystal. We can therefore conclude that for a protein with a low level of conformational dynamics, and thus a very narrow distribution of conformations in solution and in the crystal, we can extract very precise backbone dihedral angle constraints.

Acknowledgements Work in the SG laboratory is funded by The Institute of Cancer Research (ICR), by Cancer Research UK through a Career Establishment Award to SG (C47521/A16217) and by the Lister Institute of Preventive Medicine. Work in the IC laboratory is supported by ICR and Cancer Research UK through funding to the Cancer Therapeutics Unit (C309/A11566). KP was supported by a Wellcome Trust Ph.D. studentship (WT102360/Z/13/Z). SG and IC acknowledge funding through a Faringdon Proof of Concept Fund Award from ICR. NMR spectra were recorded in the King's College London Centre of Biomolecular Spectroscopy and at the MRC Biomolecular NMR Centre based at the Francis Crick Institute. The authors wish to thank Andrew Atkinson and Geoff Kelly for help with recording the spectra and Maggie Liu for initial NMR work and advice.

Open Access This article is distributed under the terms of the Creative Commons Attribution 4.0 International License (http://creativeco mmons.org/licenses/by/4.0/), which permits unrestricted use, distribution, and reproduction in any medium, provided you give appropriate credit to the original author(s) and the source, provide a link to the Creative Commons license, and indicate if changes were made.

\section{References}

Bhardwaj A, Yang Y, Ueberheide B, Smith S (2017) Whole proteome analysis of human tankyrase knockout cells reveals targets of tankyrase-mediated degradation. Nat Commun 8:2214. https:// doi.org/10.1038/s41467-017-02363-w

Chang P, Coughlin M, Mitchison TJ (2005) Tankyrase-1 polymerization of poly(ADP-ribose) is required for spindle structure and function. Nature Cell Biol 7:1133-1139. https://doi.org/10.1038/ ncb1322

Chang P, Coughlin M, Mitchison TJ (2009) Interaction between Poly(ADP-ribose) and NuMA contributes to mitotic spindle pole assembly. Mol Biol Cell 20:4575-4585. https://doi.org/10.1091/ mbc.e09-06-0477

Cheung M-S, Maguire ML, Stevens TJ, Broadhurst RW (2010) DANGLE: a Bayesian inferential method for predicting protein backbone dihedral angles and secondary structure. J Magn Reson 202:223-233. https://doi.org/10.1016/j.jmr.2009.11.008

Chi NW, Lodish HF (2000) Tankyrase is a golgi-associated mitogenactivated protein kinase substrate that interacts with IRAP in GLUT4 vesicles. J Biol Chem 275:38437-38444. https://doi. org/10.1074/jbc.M007635200

DaRosa PA, Wang Z, Jiang X et al (2015) Allosteric activation of the RNF146 ubiquitin ligase by a poly(ADP-ribosyl)ation signal. Nature 517:223-226. https://doi.org/10.1038/nature13826

DaRosa PA, Klevit RE, Xu W (2018) Structural basis for tankyraseRNF146 interaction reveals noncanonical tankyrase-binding motifs. Protein Sci 27:1057-1067. https://doi.org/10.1002/ pro.3413

De Rycker M, Price CM (2004) Tankyrase polymerization is controlled by its sterile alpha motif and poly(ADP-ribose) polymerase domains. Mol Cell Biol 24:9802-9812. https://doi.org/10.1128/ MCB.24.22.9802-9812.2004

Dynek JN, Smith S (2004) Resolution of sister telomere association is required for progression through mitosis. Science 304:97-100. https://doi.org/10.1126/science.1094754 
Eisemann T, McCauley M, Langelier M-F et al (2016) Tankyrase-1 ankyrin repeats form an adaptable binding platform for targets of ADP-ribose modification. Structure 24:1679-1692. https://doi. org/10.1016/j.str.2016.07.014

Guettler S, LaRose J, Petsalaki E et al (2011) Structural basis and sequence rules for substrate recognition by tankyrase explain the basis for cherubism disease. Cell 147:1340-1354. https://doi. org/10.1016/j.cell.2011.10.046

Haikarainen T, Krauss S, Lehtiö L (2014) Tankyrases: structure, function and therapeutic implications in cancer. Curr Pharm Des 20:6472-6488. https://doi.org/10.2174/13816128206661406301 01525

Hottiger MO, Hassa PO, Lüscher B et al (2010) Toward a unified nomenclature for mammalian ADP-ribosyltransferases. Trends Biochem Sci 35:208-219. https://doi.org/10.1016/j. tibs.2009.12.003

Huang S-MA, Mishina YM, Liu S et al (2009) Tankyrase inhibition stabilizes axin and antagonizes Wnt signalling. Nature 461:614 620. https://doi.org/10.1038/nature08356

Jia J, Qiao Y, Pilo MG et al (2017) Tankyrase inhibitors suppress hepatocellular carcinoma cell growth via modulating the Hippo cascade. PLoS ONE 12:e184068. https://doi.org/10.1371/journ al.pone. 0184068

Lehtiö L, Collins R, van den Berg S et al (2008) Zinc binding catalytic domain of human tankyrase 1. J Mol Biol 379:136-145. https:// doi.org/10.1016/j.jmb.2008.03.058

Li B, Qiao R, Wang Z et al (2016) Crystal structure of a tankyrase 1-telomere repeat factor 1 complex. Acta Crystallogr F Struct Biol Commun 72:320-327. https://doi.org/10.1107/S2053230X16004131

Li X, Han H, Zhou M-T et al (2017) Proteomic analysis of the human tankyrase protein interaction network reveals its role in pexophagy. Cell Rep 20:737-749. https://doi.org/10.1016/j.celre p.2017.06.077

Lupo B, Vialard J, Sassi F et al (2016) Tankyrase inhibition impairs directional migration and invasion of lung cancer cells by affecting microtubule dynamics and polarity signals. BMC Biol 14:5. https://doi.org/10.1186/s12915-016-0226-9

Mariotti L, Templeton CM, Ranes M et al (2016) Tankyrase requires SAM domain-dependent polymerization to support Wnt-betacatenin signaling. MolCell 63:498-513. https://doi.org/10.1016/j. molcel.2016.06.019

Mariotti L, Pollock K, Guettler S (2017) Regulation of Wnt/betacatenin signalling by tankyrase-dependent poly(ADP-ribosyl) ation and scaffolding. Br J Pharmacol 174:4611-4636. https:// doi.org/10.1111/bph.14038

Marley J, Lu M, Bracken C (2001) A method for efficient isotopic labeling of recombinant proteins. J Biomol NMR 20:71-75

Morrone S, Cheng Z, Moon RT et al (2012) Crystal structure of a Tankyrase-Axin complex and its implications for Axin turnover and Tankyrase substrate recruitment. Proc Natl Acad Sci USA 109:1500-1505. https://doi.org/10.1073/pnas.1116618109

Mosavi LK, Minor DL, Peng Z-Y (2002) Consensus-derived structural determinants of the ankyrin repeat motif. Proc Natl Acad Sci USA 99:16029-16034. https://doi.org/10.1073/pnas.252537899

Nagy Z, Kalousi A, Furst A et al (2016) Tankyrases promote homologous recombination and check point activation in response to DSBs. PLoS Genet 12:e1005791. https://doi.org/10.1371/journ al.pgen.1005791

Pollock K, Ranes M, Collins I, Guettler S (2017) Identifying and validating tankyrase binders and substrates: a candidate approach. Methods MolBiol 1608:445-473. https://doi. org/10.1007/978-1-4939-6993-7_28

Preimesberger MR, Majumdar A, Aksel T et al (2015) Direct NMR detection of bifurcated hydrogen bonding in the $\alpha$-helix N-caps of ankyrin repeat proteins. JAmChemSoc 137:1008-1011. https ://doi.org/10.1021/ja510784g
Riccio AA, McCauley M, Langelier M-F, Pascal JM (2016) Tankyrase sterile $\alpha$ motif domain polymerization is required for its role in Wnt signaling. Structure 24:1573-1581. https://doi.org/10.1016/j. str.2016.06.022

Riffell JL, Lord CJ, Ashworth A (2012) Tankyrase-targeted therapeutics: expanding opportunities in the PARP family. Nat Rev Drug Discov 11:923-936. https://doi.org/10.1038/nrd3868

Sbodio JI, Chi N-W (2002) Identification of a tankyrase-binding motif shared by IRAP, TAB182, and human TRF1 but not mouse TRF1. NuMA contains this RXXPDG motif and is a novel tankyrase partner. J Biol Chem 277:31887-31892. https://doi.org/10.1074/ jbc.M203916200

Seimiya H, Muramatsu Y, Smith S, Tsuruo T (2004) Functional subdomain in the ankyrin domain of tankyrase 1 required for poly(ADP-ribosyl)ation of TRF1 and telomere elongation. Mol Cell Biol 24:1944-1955. https://doi.org/10.1128/ MCB.24.5.1944-1955.2004

Smith S, de Lange T (2000) Tankyrase promotes telomere elongation in human cells. Curr Biol 10:1299-1302. https://doi.org/10.1016/ S0960-9822(00)00752-1

Smith S, Giriat I, Schmitt A, de Lange T (1998) Tankyrase, a poly(ADP-ribose) polymerase at human telomeres. Science 282:1484-1487

Troilo A, Benson EK, Esposito D et al (2016) Angiomotin stabilization by tankyrase inhibitors antagonizes constitutive TEAD-dependent transcription and proliferation of human tumor cells with Hippo pathway core component mutations. Oncotarget 7:28765-28782. https://doi.org/10.18632/oncotarget.9117

Vranken WF, Boucher W, Stevens TJ et al (2005) The CCPN data model for NMR spectroscopy: development of a software pipeline. Proteins 59:687-696. https://doi.org/10.1002/prot.20449

Vyas S, Matic I, Uchima L et al (2014) Family-wide analysis of poly(ADP-ribose) polymerase activity. Nat Commun 5:4426. https ://doi.org/10.1038/ncomms5426

Wang W, Li N, Li X et al (2015) Tankyrase inhibitors target YAP by stabilizing angiomotin family proteins. Cell Rep 13:524-532. https://doi.org/10.1016/j.celrep.2015.09.014

Wishart DS, Sykes BD (1994) The C-13 chemical-shift index-a simple method for the identification of protein secondary structure using C-13 chemical-shift data. J Biomol NMR 4:171-180

$\mathrm{Xu} \mathrm{D}$, Liu J, Fu T et al (2017a) USP25 regulates Wnt signaling by controlling the stability of tankyrases. Genes Dev 31:1024-1035. https://doi.org/10.1101/gad.300889.117

Xu W, Lau YH, Fischer G et al (2017b) Macrocyclized extended peptides: inhibiting the substrate-recognition domain of tankyrase. J Am Chem Soc 139:2245-2256. https://doi.org/10.1021/ jacs.6b10234

Yang E, Tacchelly-Benites O, Wang Z et al (2016) Wnt pathway activation by ADP-ribosylation. Nat Commun 7:11430. https://doi. org/10.1038/ncomms11430

Yeh T-YJ, Sbodio JI, Tsun Z-Y et al (2007) Insulin-stimulated exocytosis of GLUT4 is enhanced by IRAP and its partner tankyrase. Biochem J 402:279-290. https://doi.org/10.1042/BJ20060793

Zhang Y, Liu S, Mickanin C et al (2011) RNF146 is a poly(ADPribose)-directed $\mathrm{E} 3$ ligase that regulates axin degradation and $\mathrm{Wnt}$ signalling. Nat Cell Biol 13:623-629. https://doi.org/10.1038/ ncb2222

Zhong L, Ding Y, Bandyopadhyay G et al (2016) The PARsylation activity of tankyrase in adipose tissue modulates systemic glucose metabolism in mice. Diabetologia 59:582-591. https://doi. org/10.1007/s00125-015-3815-1

Publisher's Note Springer Nature remains neutral with regard to jurisdictional claims in published maps and institutional affiliations. 